\title{
POINTWISE REGULARITY FOR SOLUTIONS OF DOUBLE OBSTACLE PROBLEMS ON METRIC SPACES
}

\author{
ZOHRA FARNANA
}

\begin{abstract}
We study continuity at a given point for solutions of double obstacle problems. We obtain pointwise continuity of the solutions for discontinuous obstacles. We also show Hölder continuity for solutions of the double obstacle problems if the obstacles are Hölder continuous.
\end{abstract}

\section{Introduction}

The objective of this paper is to study continuity at a given point $x_{0}$ of the solution of the obstacle problem on certain metric spaces. The setting considered here is that of a complete metric space $X$ endowed with a metric $d$ and a Borel measure $\mu$ which is doubling, i.e., there exists a constant $C>0$ such that for all balls $B=B(x, r):=\{y \in X: d(x, y)<r\}$ in $X$ we have

$$
0<\mu(2 B) \leq C \mu(B)<\infty,
$$

where $\tau B=B(x, \tau r)$. The doubling property implies that $X$ is complete if and only if $X$ is proper, i.e., closed bounded sets are compact. We also require the space $X$ to support a $p$-Poincaré inequality, see Section for the definition.

Nonlinear potential theory on metric measure spaces has been studied in many papers, see for example Björn-Björn [1], [2], Björn-Björn-Shanmugalingam [3], [4], J. Björn [7], Cheeger [8], Heinonen-Koskela [13], KinnunenMartio [16], Koskela-MacManus [19] and Shanmugalingam [23], [24].

There are several notions of Sobolev spaces in metric spaces; see for example Cheeger [8], Hajłasz [11] and Shanmugalingam [23], [24]. We shall follow the definition of Shanmugalingam [23], where Sobolev type spaces $\mathbf{N}^{1, p}(X)$ (called the Newtonian spaces) were defined as the collection of $p$ integrable functions with $p$-integrable upper gradients. The notion of upper gradients was introduced by Heinonen-Koskela [13], as a substitute for the modulus of the usual gradient. Koskela-MacManus [19] extended the concept

Received 27 April 2010. 
to $p$-weak upper gradients. Variational inequalities can then be used to define $p$-harmonic functions as minimizers of the variational integral

$$
\int g_{u}^{p} d \mu
$$

where $g_{u}$ denotes the minimal $p$-weak upper gradient of $u$, whose existence and uniqueness were proved in Shanmugalingam [23]. The existence and uniqueness of minimizers of (1) were shown in Shanmugalingam [24].

Let $1<p<\infty$ and let $\Omega$ be a bounded open subset of $X$ whose complement has positive capacity. We minimize the variational integral (1) on $\Omega$ among all functions which have prescribed boundary values $f$ and lie between two given obstacles $\psi_{1}$ and $\psi_{2}$. The minimizer is called a solution of the $\mathscr{K}_{\psi_{1}, \psi_{2}, f}$-problem. The Dirichlet problem (the obstacle problem with $\psi_{1}=-\infty$ and $\psi_{2}=\infty$ ) for $p$-harmonic functions on metric spaces was studied e.g. in Björn-Björn [1], [2], Björn-Björn-Shanmugalingam [3], [4], Kinnunen-Shanmugalingam [17] and Shanmugalingam [24], [25].

In this paper we investigate the continuity at a given point $x_{0}$ of the solutions of the double obstacle problem. The obstacles in this context are to be regarded as quite general and irregular. In particular, they may be discontinuous. We show that if the obstacles are not continuous, but satisfy a Wiener type regularity condition, the solution is still continuous. This extends Theorem 5.4 from Malý-Ziemer [21] to metric spaces.

Although in $\mathrm{R}^{n} p$-harmonic functions are Lipschitz they need not be so in the general setting of metric spaces, see p. 149 in Koskela-Rajala-Shanmugalingam [20]. Therefore, as $p$-harmonic functions are solutions of special obstacle problems we can expect at most Hölder regularity of our solutions. Indeed, we show that the continuous solution of the single obstacle problem, with locally Hölder continuous obstacle, is locally Hölder continuous. For the double obstacle problem we prove that if the obstacles are locally Hölder continuous, then the continuous solution $u$ is Hölder continuous at every point $x_{0} \in \Omega$. This does not directly imply that $u$ is locally Hölder continuous as Example 4.2 shows.

Hölder continuity of $p$-harmonic functions on metric spaces was obtained in Kinnunen-Shanmugalingam [17]. In the Euclidean case Hölder continuity for solutions of the obstacle problems, with Hölder continuous obstacles, was obtained in Kilpeläinen-Ziemer [14] and Michael-Ziemer [22].

\section{Notation and preliminaries}

A nonnegative Borel function $g$ is said to be an upper gradient of an extended real-valued function $f$ on $X$ if for all rectifiable curves $\gamma:\left[0, l_{\gamma}\right] \rightarrow X$ 
parameterized by arc length $d s$, we have

$$
\left|f(\gamma(0))-f\left(\gamma\left(l_{\gamma}\right)\right)\right| \leq \int_{\gamma} g d s
$$

whenever both $f(\gamma(0))$ and $f\left(\gamma\left(l_{\gamma}\right)\right)$ are finite, and $\int_{\gamma} g d s=\infty$ otherwise.

If $g$ is a nonnegative measurable function on $X$ and if (2) holds for $p$-almost every curve, then $g$ is a $p$-weak upper gradient of $f$.

By saying that (2) holds for $p$-almost every curve we mean that it fails only for a curve family with zero $p$-modulus, see Definition 2.1 in Shanmugalingam [23]. If $f$ has an upper gradient in $L^{p}(X)$, then it has a minimal p-weak upper gradient $g_{f} \in L^{p}(X)$ in the sense that for every $p$-weak upper gradient $g \in L^{p}(X)$ of $f, g_{f} \leq g$ a.e., see Corollary 3.7 in Shanmugalingam [24].

We shall use the following definition of Sobolev type spaces which is equivalent to the one introduced in Shanmugalingam [23].

Definition 2.1. Let $u \in L^{p}(X)$, then we define

$$
\|u\|_{\mathbf{N}^{1, p}(X)}=\left(\int_{X}|u|^{p} d \mu+\int_{X} g_{u}^{p} d \mu\right)^{1 / p},
$$

where $g_{u}$ is the minimal $p$-weak upper gradient of $u$. The Newtonian space on $X$ is the quotient space

$$
\mathbf{N}^{1, p}(X)=\left\{u:\|u\|_{\mathbf{N}^{1, p}(X)}<\infty\right\} / \sim,
$$

where $u \sim v$ if and only if $\|u-v\|_{\mathbf{N}^{1, p}(X)}=0$.

The space $\mathbf{N}^{1, p}(X)$ is a Banach space and a lattice, see Theorem 3.7 and p. 249 in Shanmugalingam [23].

The capacity of a set $E \subset X$ is defined by

$$
C_{p}(E)=\inf _{u}\|u\|_{\mathbf{N}^{1, p}(X)}^{p},
$$

where the infimum is taken over all $u \in \mathbf{N}^{1, p}(X)$ such that $u \geq 1$ on $E$.

We say that a property holds quasieverywhere (q.e.) in $X$, if it holds everywhere except on a set of capacity zero. Newtonian functions are well defined up to sets of capacity zero, i.e., if $u, v \in \mathbf{N}^{1, p}(X)$ then $u \sim v$ if and only if $u=v$ q.e. Moreover, Corollary 3.3 in Shanmugalingam [23] shows that if $u, v \in \mathbf{N}^{1, p}(X)$ and $u=v$ a.e., then $u=v$ q.e.

From now on we assume that $X$ supports a $p$-Poincaré inequality i.e., there exist constants $C>0$ and $\lambda \geq 1$ such that for all balls $B(z, r)$ in $X$, all 
integrable functions $u$ on $X$ and all upper gradients $g$ of $u$ we have

$$
f_{B(z, r)}\left|u-u_{B(z, r)}\right| d \mu \leq C r\left(f_{B(z, \lambda r)} g^{p} d \mu\right)^{1 / p},
$$

where $u_{B(z, r)}:=f_{B(z, r)} u d \mu:=\mu(B(z, r))^{-1} \int_{B(z, r)} u d \mu$.

Under the above assumptions, every function $u \in \mathbf{N}^{1, p}(X)$ is quasicontinuous, i.e., for every $\varepsilon>0$ there is an open set $G \subset X$ such that $C_{p}(G)<\varepsilon$ and $\left.u\right|_{X \backslash G}$ is continuous, see Theorem 1.1 in Björn-Björn-Shanmugalingam [5]. Moreover, when restricted to $\mathrm{R}^{n}$, the Newtonian space $\mathbf{N}^{1, p}\left(\mathrm{R}^{n}\right)$ is the refined Sobolev space $W^{1, p}\left(\mathbf{R}^{n}\right)$, as defined in Chapter 4 in Heinonen-KilpeläinenMartio [12].

We say that $f \in \mathbf{N}_{\text {loc }}^{1, p}(\Omega)$ if $f \in \mathbf{N}^{1, p}\left(\Omega^{\prime}\right)$ for every $\Omega^{\prime} \subset \subset \Omega$, where by $\Omega^{\prime} \subset \subset \Omega$ we mean that the closure of $\Omega^{\prime}$ is a compact subset of $\Omega$. Let also

$$
\mathbf{N}_{0}^{1, p}(\Omega)=\left\{\left.u\right|_{\Omega}: u \in \mathbf{N}^{1, p}(X) \text { and } u=0 \text { in } X \backslash \Omega\right\} .
$$

The following definition is slightly different from the notation used in Kinnunen-Martio [16]. We use q.e. inequalities rather than a.e. inequalities as in [16], for more discussion see p. 265 in Farnana [10].

Definition 2.2. Let $V \subset X$ be a nonempty bounded open set such that $C_{p}(X \backslash V)>0$, let $f \in \mathbf{N}^{1, p}(V)$ and $\psi_{i}: V \rightarrow \overline{\mathrm{R}}, i=1,2$. Then we define the obstacle problem with obstacles $\psi_{1}, \psi_{2}$ and boundary values $f$ by

$\mathscr{K}_{\psi_{1}, \psi_{2}, f}(V)=\left\{v \in \mathbf{N}^{1, p}(V): v-f \in \mathbf{N}_{0}^{1, p}(V)\right.$ and $\psi_{1} \leq v \leq \psi_{2}$ q.e. in $\left.V\right\}$.

Furthermore, a function $u \in \mathscr{K}_{\psi_{1}, \psi_{2}, f}(V)$ is a solution of the $\mathscr{K}_{\psi_{1}, \psi_{2}, f}(V)$ problem if

$$
\int_{V} g_{u}^{p} d \mu \leq \int_{V} g_{v}^{p} d \mu \quad \text { for all } \quad v \in \mathscr{K}_{\psi_{1}, \psi_{2}, f}(V) .
$$

We also let $\mathscr{K}_{\psi_{1}, \psi_{2}, f}=\mathscr{K}_{\psi_{1}, \psi_{2}, f}(\Omega), \mathscr{K}_{\psi, f}(V)=\mathscr{K}_{\psi, \infty, f}(V)$ and $\mathscr{K}_{\psi, f}=$ $\mathscr{K}_{\psi, f}(\Omega)$.

A function $u \in \mathbf{N}_{\text {loc }}^{1, p}(\Omega)$ is a minimizer in $\Omega$ if it is a solution of the $\mathscr{K}_{-\infty, u}\left(\Omega^{\prime}\right)$-problem for every open $\Omega^{\prime} \subset \subset \Omega$. Also $u$ is $p$-harmonic in $\Omega$ if it is a continuous minimizer. Similarly, a function $u \in \mathbf{N}_{\text {loc }}^{1, p}(\Omega)$ is a $s u$ perminimizer in $\Omega$ if it is a solution of the $\mathscr{K}_{u, u}\left(\Omega^{\prime}\right)$-problem for every open $\Omega^{\prime} \subset \subset \Omega$. In [16], Kinnunen and Martio showed that if $u$ is a solution of the $\mathscr{K}_{\psi, f}$-problem ${ }_{1}^{\prime}$, then it is a superminimizer in $\Omega$ and its lower semicontinuous regularization $u^{*}(x):=\operatorname{ess} \liminf _{y \rightarrow x} u(y)$ equals $u$ q.e. in $\Omega$ and is the 
unique lower semicontinuously regularized solution. Moreover $u^{*}$ is superharmonic, see e.g. Kinnunen-Martio [16] for the definition of superharmonic functions.

We shall need the following results from Farnana [10]. We let $u_{+}=\max \{u, 0\}$ be the positive part of $u$.

Lemma 2.3. Let $\psi, \psi^{\prime}, \phi, \phi^{\prime}: \Omega \rightarrow \overline{\mathrm{R}}$ and $f, f^{\prime} \in \mathbf{N}^{1, p}(\Omega)$. Let $u$ be a solution of the $\mathscr{K}_{\psi, \phi, f}$-problem and $u^{\prime}$ be a solution of the $\mathscr{K}_{\psi^{\prime}, \phi^{\prime}, f^{\prime}}$-problem. Assume that $\left(f-f^{\prime}\right)_{+} \in \mathbf{N}_{0}^{1, p}(\Omega)$ and that $\psi \leq \psi^{\prime}$ and $\phi \leq \phi^{\prime}$ q.e. in $\Omega$. Then $u \leq u^{\prime}$ q.e. in $\Omega$.

Lemma 2.4. Let $\psi_{i}: \Omega \rightarrow \overline{\mathrm{R}}, i=1,2$, and $f \in \mathbf{N}^{1, p}(\Omega)$. Let $u$ be a

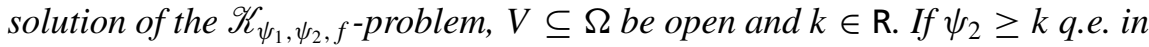
$V$, then $u_{k}=\min \{u, k\}$ is a superminimizer in $V$.

The following result, which we will need later, is a combination of Theorem 4.2 and Remark 4.4 in Kinnunen-Shanmugalingam [17] and Theorem 4.4 and Remark 4.5 in Kinnunen-Martio [16]. It shows that the solution of the single obstacle problem is locally bounded from above if the obstacle is locally bounded from above.

TheOREM 2.5. Let $B(x, 2 r) \subset \Omega$ and $k \geq \psi$ q.e. in $B(x, 2 r)$. Let $u$ be $a$ solution of the $\mathscr{K}_{\psi, f}(\Omega)$-problem. Then for all $q>0$ and $r>0$,

$$
\underset{B(x, r)}{\operatorname{ess} \sup } u \leq k+C\left(f_{B(x, 2 r)}(u-k)_{+}^{q} d \mu\right)^{1 / q} .
$$

Remark 2.6. The comparison Lemma 2.3 between a solution $u$ of the $\mathscr{K}_{\psi_{1}, \psi_{2}, f}$-problem and a solution $v$ of the $\mathscr{K}_{\psi_{1}, f}$-problem implies that $u \leq v$ q.e. in $\Omega$, hence a.e. This together with Theorem 2.5 implies that $u$ is essentially locally bounded from above if $\psi_{1}$ is essentially locally bounded from above. Similarly it follows that $u$ is essentially locally bounded from below if $\psi_{2}$ is essentially bounded from below, using that $-u$ is a solution of the $\mathscr{K}_{-\psi_{2},-\psi_{1},-f^{-}}$ problem.

\section{Continuity in the presence of irregular obstacles}

In this section we will initiate a study of the regularity of solutions of the double obstacle problems. Since we are concerned with pointwise regularity, we will consider a fixed point $x_{0} \in \Omega$ throughout the rest of this section.

The relative capacity of $E \subset \subset B$ with respect to a ball $B$ is

$$
\operatorname{Cap}_{p}(E, B)=\inf _{u} \int_{B} g_{u}^{p} d \mu,
$$


where the infimum is taken over all $u \in \mathbf{N}_{0}^{1, p}(B)$ such that $u \geq 1$ on $E$.

A set $E \subset X$ is $p$-thin at $x \in X$ if

$$
\int_{0}^{1}\left(\frac{\operatorname{Cap}_{p}(E \cap B(x, t), B(x, 2 t))}{\operatorname{Cap}_{p}(B(x, t), B(x, 2 t))}\right)^{1 /(p-1)} \frac{d t}{t}<\infty,
$$

and $G \subset X$ is $p$-finelyopen if $X \backslash G$ is $p$-thin at every $x \in G$. The complement of a $p$-finely open set is called $p$-finely closed. The $p$-fine open sets generate the $p$-fine topology which is finer than the metric topology, see J. Björn [7], Kinnunen-Latvala [15] and Korte [18]. In the Euclidean case the $p$-fine topology was studied in Malý-Ziemer [21].

Definition 3.1. A point $x_{0}$ is called a Wiener point relative to the obstacles $\psi_{1}$ and $\psi_{2}$ if

$$
\begin{gathered}
p \text {-fine } \lim _{x \rightarrow x_{0}} \sup \psi_{1}(x)=C_{p} \text { - } \limsup _{x \rightarrow x_{0}} \psi_{1}(x), \\
p \text {-fine } \lim _{x \rightarrow x_{0}} \inf \psi_{2}(x)=C_{p}-\liminf _{x \rightarrow x_{0}} \psi_{2}(x)
\end{gathered}
$$

and

$$
C_{p}-\limsup _{x \rightarrow x_{0}} \psi_{1}(x) \leq C_{p}-\liminf _{x \rightarrow x_{0}} \psi_{2}(x) .
$$

Here $C_{p}$-lim sup and $C_{p}$-lim inf stand for essential limits up to sets of capacity zero, while $p$-fine lim sup and $p$-fine lim inf stand for limits in the $p$-fine topology.

REMARK 3.2. The inequality (4) is necessary even if we just want to insert a function continuous at $x_{0}$ which lies q.e. between $\psi_{1}$ and $\psi_{2}$.

A function $u$ is $p$-finely continuous at $x_{0}$ if $u\left(x_{0}\right)=p$-fine $\limsup _{x \rightarrow x_{0}} u(x)$ $=p$-fine $\liminf \operatorname{in}_{x \rightarrow x_{0}} u(x)$. If $u \in \mathbf{N}^{1, p}(\Omega)$ then $u$ is $p$-finely continuous at q.e. $x \in \Omega$, see Theorem 4.6 in J. Björn [7].

We say that a function $u$ is $p$-finely represented if $u$ is defined everywhere in $\Omega$ and

$$
p \text {-fine } \lim _{y \rightarrow x} \inf u(y) \leq u(x) \leq p \text {-fine } \lim _{y \rightarrow x} \sup u(y)
$$

for each $x \in \Omega$. By Theorem 4.6 in J. Björn [7] every function $u \in \mathbf{N}^{1, p}(\Omega)$ can be $p$-finely represented, e.g.

$$
x \mapsto p \text {-fine } \lim _{y \rightarrow x} \inf u(y) \quad \text { and } \quad x \mapsto p \text {-fine } \lim _{y \rightarrow x} \sup u(y)
$$


are $p$-fine representatives of $u$. The following result extends Theorem 5.4 from Malý-Ziemer [21] to metric spaces. The proof below has been inspired by the proof in [21].

Theorem 3.3. Let $f \in \mathbf{N}^{1, p}(\Omega), \psi_{1}: \Omega \rightarrow[-\infty, \infty)$ and $\psi_{2}: \Omega \rightarrow$ $(-\infty, \infty]$. Let $x_{0}$ be a Wiener point relative to $\psi_{1}$ and $\psi_{2}$ and let $u$ be a $p$ -

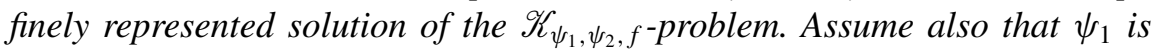
bounded from above in a neighbourhood of $x_{0}$ and $\psi_{2}$ is bounded from below in a neighbourhood of $x_{0}$. Then

$$
C_{p}-\limsup _{x \rightarrow x_{0}} \psi_{1}(x) \leq u\left(x_{0}\right) \leq C_{p}-\liminf _{x \rightarrow x_{0}} \psi_{2}(x) .
$$

Moreover $u$ is real-valued continuous at $x_{0}$.

Note that in Theorem 3.3 we allow $C_{p}$-lim $\sup _{x \rightarrow x_{0}} \psi_{1}(x)$ to take the value $-\infty$ and $C_{p}$-liminf $x_{x \rightarrow x_{0}} \psi_{2}(x)$ to take the value $\infty$.

Proof. Let

$$
\underline{u}(x)=p \text {-fine } \lim _{y \rightarrow x} \inf u(y) \quad \text { and } \quad \bar{u}(x)=p \text {-fine } \lim _{y \rightarrow x} \sup u(y) .
$$

Note that for every $k \in \mathrm{R}$ we have

$$
\begin{aligned}
& p \text {-fine } \lim _{y \rightarrow x} \inf (\min \{u, k\})(y)=\min \{\underline{u}, k\}(x)=: \underline{u}_{k}(x), \\
& p \text {-fine } \lim _{y \rightarrow x} \sup (\min \{u, k\})(y)=\min \{\bar{u}, k\}(x)=: \bar{u}_{k}(x)
\end{aligned}
$$

and

$$
\text { ess } \lim _{y \rightarrow x} \inf (\min \{u, k\})(y)=\min \left\{u^{*}, k\right\}(x)=: u_{k}^{*}(x),
$$

where $u^{*}(x)=$ ess $\liminf _{y \rightarrow x} u(y)$.

Next, as $u$ is $p$-finely represented we have

$$
\underline{u} \leq u \leq \bar{u} \quad \text { everywhere in } \Omega .
$$

Choose $k<C_{p}-\lim \inf _{x \rightarrow x_{0}} \psi_{2}(x)$. Then there exists $r>0$ such that $B\left(x_{0}, r\right)$ $\subset \Omega, \psi_{2}$ is bounded from below in $B\left(x_{0}, r\right)$ and $k<\psi_{2}(x)$ for q.e. $x \in$ $B\left(x_{0}, r\right)$. It follows that $u_{k}:=\min \{u, k\}$ is a superminimizer in $B\left(x_{0}, r\right)$, by Lemma 2.4. Then the lower semicontinuous regularization $u_{k}^{*}$ of $u_{k}$ is a superharmonic function and hence $p$-finely continuous by Theorem 4.4 in J. Björn [7] (or Theorem 4.3 in Korte [18]). This and the fact that $u_{k}^{*}=u_{k}$ q.e. 
in $\Omega$ imply that, for $x \in B\left(x_{0}, r\right)$,

$$
\begin{aligned}
\underline{u}_{k}(x) & =p \text {-fine } \lim _{y \rightarrow x} \inf u_{k}(y)=p \text {-fine } \liminf _{y \rightarrow x} u_{k}^{*}(y)=u_{k}^{*}(x) \\
& =p \text {-fine } \lim _{y \rightarrow x} \sup u_{k}^{*}(y)=p \text {-fine } \underset{y \rightarrow x}{\lim \sup } u_{k}(y)=\bar{u}_{k}(x) .
\end{aligned}
$$

This and (6) show that $u_{k}=u_{k}^{*}$ everywhere in $B\left(x_{0}, r\right)$. Hence $u_{k}$ is both lower semicontinuous and $p$-finely continuous at $x_{0}$, whenever $k<C_{p}$-lim inf $x_{x \rightarrow x_{0}}$ $\psi_{2}(x)$. As for $k=k_{0}:=C_{p}-\liminf \operatorname{in}_{x \rightarrow x_{0}} \psi_{2}(x)<\infty$ we have $u_{k} \nearrow u_{k_{0}}$ uniformly as $k \nearrow k_{0}$. Then for every $\varepsilon>0$,

$$
u_{k_{0}}\left(x_{0}\right) \leq u_{k_{0}-\varepsilon}\left(x_{0}\right)+\varepsilon \leq \liminf _{x \rightarrow x_{0}} u_{k_{0}-\varepsilon}(x)+\varepsilon \leq \liminf _{x \rightarrow x_{0}} u_{k_{0}}(x)+\varepsilon .
$$

Since $\varepsilon>0$ was arbitrary this shows that $u_{k_{0}}$ is lower semicontinuous at $x_{0}$. Similarly we conclude that $u_{k_{0}}$ is $p$-finely continuous at $x_{0}$, using that $u_{k}$ is $p$-finely continuous for all $k<k_{0}$ and that $u_{k} \nearrow u_{k_{0}}$ uniformly in $\Omega$, as $k \nearrow k_{0}$.

Let $k$ be a real number with

$$
C_{p}-\limsup _{x \rightarrow x_{0}} \psi_{1}(x) \leq k \leq C_{p}-\liminf _{x \rightarrow x_{0}} \psi_{2}(x) .
$$

Then we obtain

$$
\begin{aligned}
u\left(x_{0}\right) & \geq u_{k}\left(x_{0}\right)=p \text {-fine } \lim _{x \rightarrow x_{0}} u_{k}(x) \\
& \geq p \text {-fine } \lim _{x \rightarrow x_{0}} \sup \psi_{1}(x)=C_{p} \text { - } \limsup _{x \rightarrow x_{0}} \psi_{1}(x) .
\end{aligned}
$$

Similarly, as $-u$ is a $p$-finely represented solution of the $\mathscr{K}_{-\psi_{2},-\psi_{1},-f}$-problem, we have that $u\left(x_{0}\right) \leq C_{p}$-lim inf $x_{x \rightarrow x_{0}} \psi_{2}(x)$ and hence (5) is established.

Next, we show that $u$ is real-valued at $x_{0}$. As $\psi_{1}$ is bounded from above in a neighbourhood of $x_{0}$, there exist $r>0$ and $M \in \mathrm{R}$ such that $\psi_{1} \leq M$ in $B=B\left(x_{0}, r\right)$. Applying Remark 2.6 to the ball $B$ implies that $u \leq M^{\prime}<\infty$ q.e. in $B$ for some $M^{\prime} \in \mathrm{R}$. Since $u$ is $p$-finely represented we have $u \leq \bar{u} \leq$ $M^{\prime}<\infty$ everywhere in $B$. Similarly we get that $u>-\infty$ in a neighbourhood of $x_{0}$.

Finally we show that $u$ is continuous at $x_{0}$. As $u$ is real-valued at $x_{0}$ we can take $k=u\left(x_{0}\right) \leq C_{p}$-liminf $\operatorname{in}_{x \rightarrow x_{0}} \psi_{2}(x)$. Then $u_{k}$ is lower semicontinuous at $x_{0}$, by the the first part of the proof. It then follows that

$$
u\left(x_{0}\right)=u_{k}\left(x_{0}\right) \leq \liminf _{x \rightarrow x_{0}} u_{k}(x) \leq \liminf _{x \rightarrow x_{0}} u(x) .
$$


Hence $u$ is lower semicontinuous at $x_{0}$. Using this and the fact that $-u$ is a $p$-finely represented solution of the $\mathscr{K}_{-\psi_{2},-\psi_{1},-f}$-problem, we get that

$$
\begin{aligned}
u\left(x_{0}\right) & =-(-u)\left(x_{0}\right) \geq-\liminf _{x \rightarrow x_{0}}(-u)(x) \\
& =\limsup _{x \rightarrow x_{0}} u(x) \geq \liminf _{x \rightarrow x_{0}} u(x) \geq u\left(x_{0}\right),
\end{aligned}
$$

which shows the continuity of $u$ at $x_{0}$.

From Theorem 3.3 we get the following immediate corollary. It extends Theorem 3.9 from Farnana [10] where the continuity of $u$ in $\Omega$ was proved for real-valued continuous obstacles.

Corollary 3.4. Let $\psi_{1}: \Omega \rightarrow[-\infty, \infty)$ and $\psi_{2}: \Omega \rightarrow(-\infty, \infty]$ be continuous (as $\overline{\mathrm{R}}$-valued functions) at $x_{0} \in \Omega$. Let $u$ be a p-finely represented

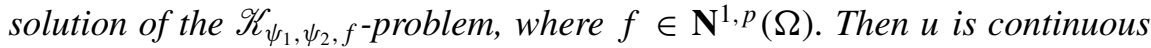
at $x_{0}$ (as a real-valued function). In particular if $\psi_{1}$ and $\psi_{2}$ are extended real-valued continuous on $\Omega$, then $u$ is real-valued continuous on $\Omega$.

\section{Hölder continuity of the solutions}

It was shown by Kinnunen-Shanmugalingam [17] that $p$-harmonic functions are locally Hölder continuous. In this section we extend this result to obstacle problems with Hölder continuous obstacles. Similar results in $\mathrm{R}^{n}$ have been obtained by Kilpeläinen-Ziemer [14] and Michael-Ziemer [22]. Although, in $\mathrm{R}^{n}, p$-harmonic functions are Lipschitz they need not be so in the general setting of metric spaces, see p. 149 in Koskela-Rajala-Shanmugalingam [20].

We shall need the following result, for a proof see Theorem 9.2 in A. BjörnMarola [6].

THEOREM 4.1. If $u$ is a nonnegative superminimizer in $\Omega$, then there are $q>0$ and $C>0$ independent of $u$ such that

$$
\left(f_{B(x, 2 r)} u^{q} d \mu\right)^{1 / q} \leq C \underset{B(x, r)}{\operatorname{essinf}} u
$$

for every ball $B(x, r)$ such that $B(x, 20 \lambda r) \subset \Omega$.

Here $\lambda$ is the dilation constant in the Poincare inequality.

We say that $f$ is Hölder continuous at $x_{0}$ if there exist $r>0, \alpha>0$ and $C>0$ such that, for all $x \in B\left(x_{0}, r\right)$, we have

$$
\left|f(x)-f\left(x_{0}\right)\right| \leq C d\left(x, x_{0}\right)^{\alpha} .
$$


We also say that $f$ is locally Hölder continuous in $\Omega$ if for each $x_{0} \in \Omega$ there exist $r>0, \alpha>0$ and $C>0$ (depending on $x_{0}$ ) such that for all $x, y \in B\left(x_{0}, r\right)$, we have

$$
|f(x)-f(y)| \leq C d(x, y)^{\alpha} .
$$

Note that Hölder continuity of $f$ at every $x_{0} \in \Omega$ does not imply, in general, that $f$ is locally Hölder continuous as the following example shows.

Example 4.2. The function

$$
f(x)= \begin{cases}x \sin e^{1 / x}, & x>0 \\ 0, & x \leq 0\end{cases}
$$

is continuously differentiable in $\mathrm{R} \backslash\{0\}$. Hence it is Hölder continuous (even Lipschitz continuous) at every point in $\mathrm{R} \backslash\{0\}$. As $|f(x)| \leq|x|$ at every $x \in \mathrm{R}$ we also have that $f$ is Hölder continuous at $x=0$. Thus $f$ is Hölder continuous at every $x \in \mathrm{R}$. But $f$ is not Hölder continuous in any neighbourhood of zero. If $f$ were Hölder continuous in a neighbourhood of zero then there would exist $\varepsilon>0, C>0$ and $\alpha>0$ such that

$$
|f(x)-f(y)| \leq C|x-y|^{\alpha} \quad \text { for all } \quad x, y \in B(0, \varepsilon) .
$$

Let

$$
x=\frac{1}{\log \left(2 \pi n+\frac{\pi}{2}\right)} \quad \text { and } \quad y=\frac{1}{\log \left(2 \pi n-\frac{\pi}{2}\right)}
$$

for $n \in \mathrm{N}$ sufficiently large so that $0<x<y<\varepsilon$. Then we have

$$
|f(x)-f(y)|=x+y>y>\frac{1}{\log (2 \pi n)} .
$$

On the other hand we have

$$
\begin{aligned}
|x-y|=y-x & =\frac{\log \left(2 \pi n+\frac{\pi}{2}\right)-\log \left(2 \pi n-\frac{\pi}{2}\right)}{\log \left(2 \pi n+\frac{\pi}{2}\right) \log \left(2 \pi n-\frac{\pi}{2}\right)} \\
& \leq 2 \frac{\log \left(1+\frac{1}{4 n}\right)-\log \left(1-\frac{1}{4 n}\right)}{(\log (2 \pi n))^{2}} \\
& \leq \frac{2}{n} .
\end{aligned}
$$

It follows from (7) and (8) that for sufficiently large $n$ we have

$$
|f(x)-f(y)| \geq \frac{1}{\log (2 \pi n)}>C\left(\frac{2}{n}\right)^{\alpha} \geq C|x-y|^{\alpha},
$$


a contradiction. Hence $f$ is not Hölder continuous in any neighbourhood of zero.

THeOREM 4.3. Let $\psi: \Omega \rightarrow \mathrm{R}$ be locally Hölder continuous and $f \in$ $\mathbf{N}^{1, p}(\Omega)$. Let $u$ be the continuous solution of the $\mathscr{K}_{\psi, f}$-problem. Then $u$ is locally Hölder continuous in $\Omega$.

Proof. Let $B=B\left(x_{0}, R\right)$ and $20 \lambda B \subset \Omega$, where $\lambda$ is the dilation constant from the $p$-Poincaré inequality, be such that $\psi$ is Hölder continuous in $2 B$, with exponent $\alpha$ and constant $C_{0}$. Let also, for $r>0$,

$$
\underline{u}(r)=\inf _{B\left(x_{0}, r\right)} u \quad \text { and } \quad \bar{u}(r)=\sup _{B\left(x_{0}, r\right)} u
$$

and similarly for $\psi$. As $u-\underline{u}(20 \lambda R)$ is a nonnegative superminimizer in $20 \lambda B$ it follows from the weak Harnack inequality, Theorem 4.1, that there are $q>0$ and $C_{1}>0$ such that

$$
\begin{aligned}
\underline{u}(R)-\underline{u}(20 \lambda R) & =\inf _{B}(u-\underline{u}(20 \lambda R)) \\
& \geq \frac{1}{C_{1}}\left(f_{2 B}(u-\underline{u}(20 \lambda R))^{q} d \mu\right)^{1 / q} .
\end{aligned}
$$

We consider two cases. First assume that $\bar{\psi}(2 R) \leq \underline{u}(20 \lambda R)$ which implies that $u(20 \lambda R) \geq \psi$ in $2 B$. The weak Harnack inequality, Theorem 2.5, then implies that, for some $C_{2}>0$, we have

$$
\bar{u}(R)-\underline{u}(20 \lambda R)=\sup _{B}(u-\underline{u}(20 \lambda R)) \leq C_{2}\left(f_{2 B}(u-\underline{u}(20 \lambda R))^{q} d \mu\right)^{1 / q} .
$$

This and (9) imply that

$$
\bar{u}(R)-\underline{u}(20 \lambda R) \leq C_{1} C_{2}(\underline{u}(R)-\underline{u}(20 \lambda R)) .
$$

Next suppose that $\bar{\psi}(2 R)>\underline{u}(20 \lambda R)$. It follows that

$$
(u-\bar{\psi}(2 R))_{+} \leq(u-\underline{u}(20 \lambda R))_{+}=u-\underline{u}(20 \lambda R), \quad \text { in } 20 \lambda B .
$$

The weak Harnack inequality, Theorem 2.5 , then imply that

$$
\begin{aligned}
\bar{u}(R)-\bar{\psi}(2 R) & \leq C_{2}\left(f_{2 B}(u-\bar{\psi}(2 R))_{+}^{q} d \mu\right)^{1 / q} \\
& \leq C_{2}\left(f_{2 B}(u-\underline{u}(20 \lambda R))^{q} d \mu\right)^{1 / q},
\end{aligned}
$$


for some $C_{2}>0$. This and (9) give

$$
\bar{u}(R)-\bar{\psi}(2 R) \leq C_{1} C_{2}(\underline{u}(R)-\underline{u}(20 \lambda R)) .
$$

As $\psi$ is $\alpha$-Hölder continuous in $2 B$ we have $\underline{u}(2 R) \geq \psi(2 R) \geq \bar{\psi}(2 R)-$ $C_{0}(2 R)^{\alpha}$. Then it follows that

$$
\begin{aligned}
\bar{u}(R)-\underline{u}(20 \lambda R) & =\bar{u}(R)-\underline{u}(2 R)+\underline{u}(2 R)-\underline{u}(20 \lambda R) \\
& \leq \bar{u}(R)-\underline{\psi}(2 R)+\underline{u}(R)-\underline{u}(20 \lambda R) \\
& \leq \bar{u}(R)-\bar{\psi}(2 R)+C_{0}(2 R)^{\alpha}+\underline{u}(R)-\underline{u}(20 \lambda R) \\
& \leq\left(C_{1} C_{2}+1\right)(\underline{u}(R)-\underline{u}(20 \lambda R))+C_{0}(2 R)^{\alpha} .
\end{aligned}
$$

So in both cases we have, with $C_{3}=C_{1} C_{2}+1$,

$$
\bar{u}(R)-\underline{u}(20 \lambda R) \leq C_{3}(\underline{u}(R)-\underline{u}(20 \lambda R))+C_{0}(2 R)^{\alpha} .
$$

Now, suppose that

$$
\underline{u}(R)-\underline{u}(20 \lambda R) \leq \frac{1}{1+C_{3}}(\bar{u}(R)-\underline{u}(20 \lambda R)) .
$$

We use this and (11) together with the fact that $\underline{u}(R) \geq \underline{u}(20 \lambda R)$ and $\bar{u}(R) \leq$ $\bar{u}(20 \lambda R)$ to conclude that

$$
\begin{aligned}
\bar{u}(R)-\underline{u}(R) & \leq C_{3}(\underline{u}(R)-\underline{u}(20 \lambda R))+C_{0}(2 R)^{\alpha} \\
& \leq \frac{C_{3}}{1+C_{3}}(\bar{u}(20 \lambda R)-\underline{u}(20 \lambda R))+C_{0}(2 R)^{\alpha} .
\end{aligned}
$$

On the other hand if $\underline{u}(R)-\underline{u}(20 \lambda R)>\frac{1}{1+C_{3}}(\bar{u}(R)-\underline{u}(20 \lambda R))$, we have

$$
\begin{aligned}
\bar{u}(R)-\underline{u}(R) & =\bar{u}(R)-\underline{u}(20 \lambda R)-(\underline{u}(R)-\underline{u}(20 \lambda R)) \\
& \leq \bar{u}(R)-\underline{u}(20 \lambda R)-\frac{1}{1+C_{3}}(\bar{u}(R)-\underline{u}(20 \lambda R)) \\
& \leq \frac{C_{3}}{1+C_{3}}(\bar{u}(20 \lambda R)-\underline{u}(20 \lambda R)) .
\end{aligned}
$$

Letting $\operatorname{osc}_{\tau B} u=\sup _{\tau B} u-\inf _{\tau B} u$, we thus have in both cases that

$$
\underset{B}{\operatorname{osc}} u \leq \gamma \underset{20 \lambda B}{\operatorname{osc}} u+C_{0}(2 R)^{\alpha},
$$

where $\gamma=\frac{C_{3}}{1+C_{3}}<1$ is independent of $R$ and $\alpha$. To complete the proof we iterate inequality (13). Choose $0<R<1 / 2$ and

$$
0<\alpha^{\prime}<\min \{\alpha,-\log \gamma / \log 20 \lambda\} .
$$


Then, we get that $(20 \lambda)^{\alpha^{\prime}} \gamma<1$, which will be needed below, and that

$$
\underset{B}{\operatorname{osc}} u \leq \gamma \underset{20 \lambda B}{\operatorname{osc}} u+C_{0}(2 R)^{\alpha^{\prime}} .
$$

Let $r \in(0, R)$ be arbitrary and $j \in \mathrm{N}$ be such that $(20 \lambda)^{j-1} \leq R / r<(20 \lambda)^{j}$. Applying (14) to the balls $B_{i}=B\left(x_{0},(20 \lambda)^{i} r\right), i=0,1,2, \ldots, j-1$, we obtain

$$
\begin{aligned}
& \underset{B_{0}}{\operatorname{osc}} u \leq \gamma \underset{B_{1}}{\operatorname{osc}} u+C_{0}(2 r)^{\alpha^{\prime}} \\
& \leq \gamma^{2} \underset{B_{2}}{\operatorname{osc}} u+C_{0} 2^{\alpha^{\prime}}\left((20 \lambda)^{\alpha^{\prime}} \gamma+1\right) r^{\alpha^{\prime}} \\
& \leq \gamma^{j-1} \underset{B_{j-1}}{\operatorname{osc}} u+C_{0} 2^{\alpha^{\prime}}\left(\left((20 \lambda)^{\alpha^{\prime}} \gamma\right)^{j-2}+\cdots+1\right) r^{\alpha^{\prime}} \\
& \leq \gamma^{j-1} \underset{B_{j-1}}{\operatorname{osc}} u+\frac{C_{0} 2^{\alpha^{\prime}}}{1-(20 \lambda)^{\alpha^{\prime}} \gamma} r^{\alpha^{\prime}},
\end{aligned}
$$

where we use the fact that $(20 \lambda)^{\alpha^{\prime}} \gamma<1$. By the choice of $j$ we conclude that

$$
\begin{aligned}
\gamma^{j-1} & =(20 \lambda)^{(j-1) \log \gamma / \log 20 \lambda}=(20 \lambda)^{\alpha^{\prime \prime}}\left((20 \lambda)^{j}\right)^{-\alpha^{\prime \prime}} \\
& \leq(20 \lambda)^{\alpha^{\prime \prime}}\left(\frac{R}{r}\right)^{-\alpha^{\prime \prime}}=(20 \lambda)^{\alpha^{\prime \prime}}\left(\frac{r}{R}\right)^{\alpha^{\prime \prime}},
\end{aligned}
$$

where $\alpha^{\prime \prime}=-\log \gamma / \log 20 \lambda>0$. Hence we get, using that $r / R<1$ and $\alpha^{\prime} \leq \alpha^{\prime \prime}$,

$$
\begin{aligned}
\underset{B\left(x_{0}, r\right)}{\operatorname{osc}} u & \leq(20 \lambda)^{\alpha^{\prime \prime}}\left(\frac{r}{R}\right)^{\alpha^{\prime \prime}} \underset{B\left(x_{0}, R\right)}{\operatorname{osc}} u+\frac{C_{0} 2^{\alpha^{\prime}}}{1-(20 \lambda)^{\alpha^{\prime} \gamma}} r^{\alpha^{\prime}} \\
& \leq\left(\frac{(20 \lambda)^{\alpha^{\prime \prime}}}{R^{\alpha^{\prime}}} \underset{B\left(x_{0}, R\right)}{\operatorname{osc}} u+\frac{C_{0} 2^{\alpha^{\prime}}}{1-(20 \lambda)^{\alpha^{\prime}} \gamma}\right) r^{\alpha^{\prime}} .
\end{aligned}
$$

Now, fix $r \in \mathrm{R}$ such that $0<2 r<R<1$. Then, for $x, y \in B\left(x_{0}, r\right)$, we consider two cases. First let $r \leq d(x, y)<2 r<1$. This and (15) imply that

$$
\begin{aligned}
|u(x)-u(y)| & \leq \underset{B\left(x_{0}, r\right)}{\operatorname{osc}} u \\
& \leq\left(\frac{(20 \lambda)^{\alpha^{\prime \prime}}}{R^{\alpha^{\prime}}} \underset{B\left(x_{0}, R\right)}{\operatorname{osc}} u+\frac{C_{0} 2^{\alpha^{\prime}}}{1-(20 \lambda)^{\alpha^{\prime} \gamma}}\right) d(x, y)^{\alpha^{\prime}} .
\end{aligned}
$$

Next, if $d(x, y)<r<1$, then choose $s>0$ such that $s / 2<d(x, y)<$ $s<r<R / 2$. Then (15) with $B\left(x_{0}, r\right)$ and $B\left(x_{0}, R\right)$ replaced by $B(y, s)$ and 
$B(y, R / 2)$ implies that

$$
\begin{aligned}
|u(x)-u(y)| & \leq \underset{B(y, s)}{\operatorname{osc}} u \\
& \leq\left(\frac{(20 \lambda)^{\alpha^{\prime \prime}}}{(R / 2)^{\alpha^{\prime}}} \underset{B(y, R / 2)}{\operatorname{osc}} u+\frac{C_{0} 2^{\alpha^{\prime}}}{1-(20 \lambda)^{\alpha^{\prime} \gamma}}\right) s^{\alpha^{\prime}} \\
& \leq 2^{\alpha^{\prime}}\left(\frac{(20 \lambda)^{\alpha^{\prime \prime}}}{R^{\alpha^{\prime}}} \underset{B\left(x_{0}, R\right)}{\operatorname{osc}} u+\frac{C_{0}}{1-(20 \lambda)^{\alpha^{\prime} \gamma}}\right) s^{\alpha^{\prime}} \\
& \leq 4^{\alpha^{\prime}}\left(\frac{(20 \lambda)^{\alpha^{\prime \prime}}}{R^{\alpha^{\prime}}} \underset{B\left(x_{0}, R\right)}{\operatorname{osc}} u+\frac{C_{0}}{1-(20 \lambda)^{\alpha^{\prime} \gamma}}\right) d(x, y)^{\alpha^{\prime}} .
\end{aligned}
$$

Thus we conclude from (16) and (17) that we can find $C>0$ such that for $x, y \in B\left(x_{0}, r\right)$ we have

$$
|u(x)-u(y)| \leq C d(x, y)^{\alpha^{\prime}},
$$

where $C$ depends only on $R, \alpha^{\prime \prime}=-\log \gamma / \log 20 \lambda$, the oscillation of $u$ on $B\left(x_{0}, R\right)$ and the Hölder exponent of $\psi$. Since $x_{0} \in \Omega$ was arbitrary we see that $u$ is locally Hölder continuous in $\Omega$.

For the double obstacle problem we have the following weaker result.

Theorem 4.4. Let $\psi_{i}: \Omega \rightarrow \mathrm{R}, i=1,2$, be locally Hölder continuous and $f \in \mathbf{N}^{1, p}(\Omega)$. Let $u$ be the continuous solution of the $\mathscr{K}_{\psi_{1}, \psi_{2}, f}$-problem. Then $u$ is Hölder continuous in $\Omega$ at every $x_{0} \in \Omega$.

Note that Hölder continuity of $u$ at every $x_{0} \in \Omega$ does not imply, in general, that $u$ is locally Hölder continuous as Example 4.2 shows.

Proof. Assume first that $\psi_{1}\left(x_{0}\right)<\psi_{2}\left(x_{0}\right)$. Note that either $u\left(x_{0}\right)<$ $\psi_{2}\left(x_{0}\right)$ or $u\left(x_{0}\right)>\psi_{1}\left(x_{0}\right)$. If $u\left(x_{0}\right)<\psi_{2}\left(x_{0}\right)$ find $\varepsilon>0$ and $B=B\left(x_{0}, r\right)$ such that $u\left(x_{0}\right)+\varepsilon \leq \psi_{2}\left(x_{0}\right)-\varepsilon$ and that

$$
\sup _{B} u \leq \inf _{B} u+\varepsilon=: k \quad \text { and } \quad \inf _{B} \psi_{2} \geq \psi_{2}\left(x_{0}\right)-\varepsilon .
$$

It follows that $k \geq u$ in $B$ and that

$$
k=\inf _{B} u+\varepsilon \leq u\left(x_{0}\right)+\varepsilon \leq \psi_{2}\left(x_{0}\right)-\varepsilon \leq \inf _{B} \psi_{2} \leq \psi_{2} \quad \text { in } \quad B .
$$

Lemma 2.4 then implies that $u=\min \{u, k\}$ is a superminimizer in $B$. Thus $u$ is a solution of the $\mathscr{K}_{u, u}(B)$-problem. Two applications of the comparison Lemma 2.3 between the continuous solution of the $\mathscr{K}_{\psi_{1}, u}(B)$-problem and $u$, once as $u$ is the continuous solution of the $\mathscr{K}_{\psi_{1}, \psi_{2}, u}(B)$-problem and the other as $u$ is the solution of the $\mathscr{K}_{u, u}(B)$-problem, then implies that $u$ is the 
continuous solution of the $\mathscr{K}_{\psi_{1}, u}(B)$-problem. It follows from Theorem 4.3 that there exists a ball $B\left(x_{0}, r^{\prime}\right) \subset B$ such that

$$
|u(x)-u(y)| \leq C d(x, y)^{\alpha} \quad \text { for all } \quad x, y \in B\left(x_{0}, r^{\prime}\right) .
$$

Similarly, if $u\left(x_{0}\right)>\psi_{1}\left(x_{0}\right)$ we conclude that $-u$ is the continuous solution of the $\mathscr{K}_{-\psi_{2},-u}\left(B\left(x_{0}, s\right)\right)$-problem, for some $s>0$. Hence $u$ is Hölder continuous in $B\left(x_{0}, s^{\prime}\right)$, for some $0<s^{\prime}<s$.

Next, if $\psi_{1}\left(x_{0}\right)=\psi_{2}\left(x_{0}\right)$, then $u\left(x_{0}\right)=\psi_{1}\left(x_{0}\right)=\psi_{2}\left(x_{0}\right)$, by continuity. Fix $B=B\left(x_{0}, r\right)$ such that $\psi_{1}$ and $\psi_{2}$ are Hölder continuous in $B$. Then using that $\psi_{1}$ is Hölder continuous in $B$ we get that, for $x \in B$,

$$
u\left(x_{0}\right)-u(x) \leq \psi_{1}\left(x_{0}\right)-\psi_{1}(x) \leq C_{1} d\left(x_{0}, x\right)^{\alpha_{1}},
$$

for some $C_{1}>0$ and $\alpha_{1}>0$. Similarly, using that $\psi_{2}$ is Hölder continuous in $B$, we obtain

$$
u(x)-u\left(x_{0}\right) \leq \psi_{2}(x)-\psi_{2}\left(x_{0}\right) \leq C_{2} d\left(x_{0}, x\right)^{\alpha_{2}},
$$

for some $C_{2}>0$ and $\alpha_{2}>0$. Hence $u$ is Hölder continuous at $x_{0}$.

\section{REFERENCES}

1. Björn, A., and Björn, J., Boundary regularity for p-harmonic functions and solutions of the obstacle problem on metric spaces, J. Math. Soc. Japan 58 (2006), 1211-1232.

2. Björn, A., and Björn, J., Approximations by regular sets and Wiener solutions in metric spaces, Comment. Math. Univ. Carolin. 48 (2007), 343-355.

3. Björn, A., Björn, J., and Shanmugalingam, N., The Dirichlet problem for p-harmonic functions on metric spaces, J. Reine Angew. Math. 556 (2003), 173-203.

4. Björn, A., Björn, J., and Shanmugalingam, N., The Perron method for p-harmonic functions in metric spaces, J. Differential Equations 195 (2003), 398-429.

5. Björn, A., Björn, J., and Shanmugalingam, N., Quasicontinuity of Newton-Sobolev functions and density of Lipschitz functions on metric spaces, Houston J. Math. 34 (2008), 11971211.

6. Björn, A., and Marola, N., Moser iteration for (quasi)minimizers on metric spaces, Manuscripta Math. 121 (2006), 339-366.

7. Björn, J., Fine continuity on metric spaces, Manuscripta Math. 125 (2008), 369-381.

8. Cheeger, J., Differentiability of Lipschitz functions on metric measure spaces, Geom. Funct. Anal. 9 (1999), 428-517.

9. Dal Maso, G., Mosco, U., and Vivaldi, M. A., A pointwise regularity theory for the twoobstacle problem, Acta Math. 163 (1989), 57-107.

10. Farnana, Z., The double obstacle problem on metric spaces, Ann. Acad. Sci. Fenn. Math. 34 (2009), 261-277.

11. Hajłasz, P., Sobolev spaces on an arbitrary metric spaces, Potential Anal. 5 (1996), 403-415.

12. Heinonen, J., Kilpeläinen, T., and Martio, O., Nonlinear Potential Theory of Degenerate Elliptic Equations, 2nd ed., Dover, Mineola, NY 2006. 
13. Heinonen, J., and Koskela, P., Quasiconformal maps in metric spaces with controlled geometry, Acta Math. 181 (1998), 1-61.

14. Kilpeläinen, T., and Ziemer, W. P., Pointwise regularity of solutions to nonlinear double obstacle problems, Ark. Mat. 29 (1991), 83-106.

15. Kinnunen, J., and Latvala, V., Fine regularity of superharmonic functions on metric spaces, pp. 157-167 in: Future Trends in Geometric Function Theory RNC Workshop Jyväskylä 2003, Rep. Univ. Jyväskylä Dep. Math. Stat. 92, Univ. Jyväskylä, Jyväskylä 2003.

16. Kinnunen, J., and Martio, O., Nonlinear potential theory on metric spaces, Illinois J. Math. 46 (2002), 857-883.

17. Kinnunen, J., and Shanmugalingam, N., Regularity of quasi-minimizers on metric spaces, Manuscripta Math. 105 (2001), 401-423.

18. Korte, R., A Caccioppoli estimate and fine continuity for superminimizers on metric spaces, Ann. Acad. Sci. Fenn. Math. 33 (2008), 597-604.

19. Koskela, P., and MacManus, P., Quasiconformal mappings and Sobolev spaces, Studia Math. 131 (1998), 1-17.

20. Koskela, P., Rajala, K., and Shanmugalingam, N., Lipschitz continuity of Cheeger-harmonic functions in metric measure spaces, J. Funct. Anal. 202 (2003), 147-173.

21. Malý, J., and Ziemer, W. P., Fine Regularity of Solutions of Elliptic Partial Differential Equations, Math. Surveys and Monographs 51, Amer. Math. Soc., Providence, RI 1997.

22. Michael, J. H., and Ziemer, W. P., Interior regularity for solutions to obstacle problems, Nonlinear Anal. 10 (1986), 1427-1448.

23. Shanmugalingam, N., Newtonian spaces: An extension of Sobolev spaces to metric measure spaces, Rev. Mat. Iberoamericana 16 (2000), 243-279.

24. Shanmugalingam, N., Harmonic functions on metric spaces, Illinois J. Math. 45 (2001), $1021-1050$.

25. Shanmugalingam, N., Some convergence results for p-harmonic function on metric measure spaces, Proc. London Math. Soc. (3) 87 (2003), 226-246.

DEPARTMENT OF MATHEMATICS

LINKÖPINGS UNIVERSITET

SE-581 83 LINKÖPING

SWEDEN

E-mail: zofar@mai.liu.se 